\title{
Coupling a Mobile Hole to an Antiferromagnetic Spin Background: Transient Dynamics of a Magnetic Polaron
}

Geoffrey Ji®, ${ }^{1}$ Muqing Xu®, ${ }^{1}$ Lev Haldar Kendrick $\odot,{ }^{1}$ Christie S. Chiu $\odot,{ }^{1}$ Justus C. Brüggenjürgen $\odot,{ }^{1}$ Daniel Greif, ${ }^{1}$ Annabelle Bohrdt $\odot{ }^{2,3}$ Fabian Grusdt $\odot,{ }^{3,4}$ Eugene Demler, ${ }^{1}$ Martin Lebrat $\odot,{ }^{1}$ and Markus Greiner ${ }^{1, *}$

${ }^{1}$ Department of Physics, Harvard University, 17 Oxford Street, Cambridge, Massachusetts 02138, USA

${ }^{2}$ Department of Physics and Institute for Advanced Study, Technical University of Munich, James-Franck-Straße 1, 85748 Garching, Germany

${ }^{3}$ Munich Center for Quantum Science and Technology, Schellingstraße 4, 80799 München, Germany

${ }^{4}$ Department of Physics and Arnold Sommerfeld Center for Theoretical Physics, Ludwig Maximilian University of Munich, Theresienstraße 37, 80333 München, Germany

(Received 23 December 2020; accepted 9 March 2021; published 27 April 2021)

\begin{abstract}
Understanding the interplay between charge and spin and its effects on transport is a ubiquitous challenge in quantum many-body systems. In the Fermi-Hubbard model, this interplay is thought to give rise to magnetic polarons, whose dynamics may explain emergent properties of quantum materials such as high-temperature superconductivity. In this work, we use a cold-atom quantum simulator to directly observe the formation dynamics and subsequent spreading of individual magnetic polarons. Measuring the density- and spin-resolved evolution of a single hole in a 2D Hubbard insulator with short-range antiferromagnetic correlations reveals fast initial delocalization and a dressing of the spin background, indicating polaron formation. At long times, we find that dynamics are slowed down by the spin exchange time, and they are compatible with a polaronic model with strong density and spin coupling. Our work enables the study of out-of-equilibrium emergent phenomena in the Fermi-Hubbard model, one dopant at a time.
\end{abstract}

DOI: $10.1103 /$ PhysRevX.11.021022

Subject Areas: Atomic and Molecular Physics, Strongly Correlated Materials

\section{INTRODUCTION}

Interactions between charge carriers and magnetic excitations can drastically alter the transport properties of a many-body system. Prominent examples include the Kondo effect, colossal magnetoresistance, and heavyfermion materials, where electrical resistivity is strongly affected by electron scattering against magnetic impurities and localized spins. In the two-dimensional Hubbard model, the competition between charge delocalization, related to the nearest-neighbor tunneling energy $t$, and antiferromagnetism, set by the spin exchange energy $J$, already results in intricate dynamics at the level of a single charge excitation. This iconic problem has attracted extensive theoretical attention [1-9] and can be reinterpreted as the creation and dispersion of a magnetic polaron, a charge carrier dressed by the magnetic background with renormalized properties. Because of their out-of-equilibrium

\footnotetext{
*Corresponding author. mgreiner@g.harvard.edu

Published by the American Physical Society under the terms of the Creative Commons Attribution 4.0 International license. Further distribution of this work must maintain attribution to the author(s) and the published article's title, journal citation, and DOI.
}

character, knowing both transient and long-time dynamical properties of polarons is a fundamental step towards elucidating intriguing transport phenomena seen in strongly correlated materials.

Evidence for magnetic polarons in undoped cuprates has been seen in the form of a renormalized hole dispersion measured via photoemission experiments [10]. However, an understanding of the transient behavior of a single charge excitation is obscured in solid-state systems by the presence of phonons and inherently fast electron dynamics. Ultracold atoms offer a clean and tunable quantum simulation platform where quantum dynamics can be observed with a resolution finer than all relevant timescales [11]. Quantum gas microscopy, in particular, gives access to spin and density readout and manipulation at the single-site level, thereby enabling demonstrations of onedimensional quantum walks $[12,13]$ and, more recently, the study of spin-charge deconfinement in one-dimensional Hubbard chains [14]. Previous dynamical studies of the doped 2D Fermi-Hubbard model have focused on hydrodynamic bulk transport [15-17], and equilibrium signatures of polaronic behavior have been seen in small systems $[18,19]$.

Nonetheless, observing the competition between density and spin dynamics is experimentally challenging: 
(a)

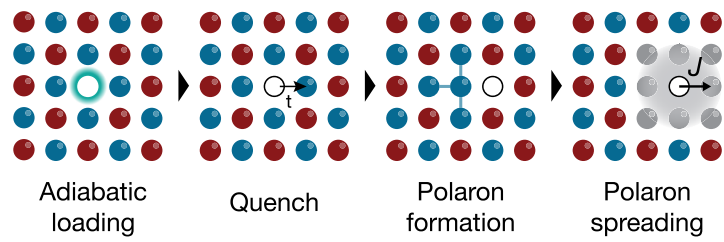

(b)

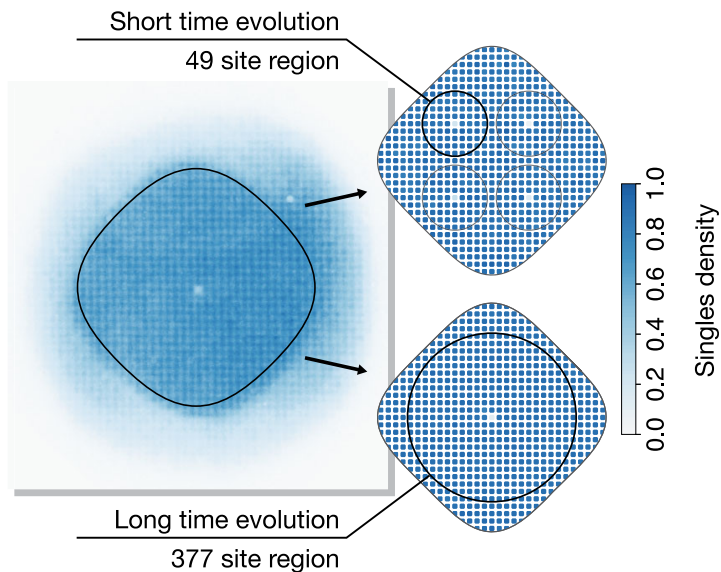

FIG. 1. Probing single-hole dynamics with a quantum gas microscope. (a) We initialize a hole in the system by adiabatically loading atoms into the lattice with a localized repulsive potential. The potential is abruptly removed, allowing the hole to delocalize at the bare tunneling rate $t$ and displace spins along its path that result in a local reversal of spin correlations compared to equilibrium. The hole is therefore locally dressed by the spin background, forming a magnetic polaron with a velocity ultimately determined by $J$. (b) Left: average of experimental images before hole release for one prepared hole. The apparent additional hole in the upper right is caused by defects in the imaging path. Right: average density of the atomic system before hole release, for one hole and four holes (to enhance the data collection rate at short evolution times). All analysis is done inside the denoted regions.

It requires simultaneously achieving low temperatures for the spin order to dramatically influence transport, and large system sizes to probe the system's evolution over long spin exchange timescales. In this work, we study the densityand spin-resolved dynamics of a single hole interacting with a two-dimensional antiferromagnetic Mott insulator of 400 sites [Fig. 1(a)]. We first observe fast dressing of the spin environment over the tunneling timescale, where quantum interference between alternative hole paths plays a large role. This fast process is followed by a slow delocalization of the hole and a slow relaxation of the spin background, which are key dynamical signatures of a magnetic polaron. We quantitatively validate our understanding of the spin dynamics by comparing them to a phenomenological model of the formation and departure of a spin polaron.

\section{EXAMINING A SINGLE DOPANT}

We prepare the initial system of fermionic lithium- 6 in a half-filled 2D square lattice with Hubbard parameters $t / \hbar=2 \pi \times 744(12) \mathrm{Hz} \quad$ and $\quad U / t=8.72(28) \quad[t / J=$ 2.18(7)] [20] at a temperature of $T / t=0.340(19)$ and with correlation length $\xi=1.695(11)$ (expressing lengths in units of the lattice spacing $a=569 \mathrm{~nm}$ and setting $k_{B}=1$ here and subsequently). Simultaneously, we project repulsive light from two digital micromirror devices (DMDs). The first DMD performs entropy redistribution and removes residual harmonic confinement over a rounded-square-shaped area of 31 sites in diameter $[21,22]$. The second DMD acts to prevent the occupation of selected sites while adiabatically loading the optical lattice, resulting in pinned holes [Fig. 1(b)]. We release the pinned holes by shutting off the light illuminating the second DMD within $0.03 \hbar / t$; then, we allow the system to evolve for a variable time $\tau$. Finally, we freeze the dynamics by rapidly increasing the lattice depth and make a projective site-resolved measurement of either the parity-projected (singles) density or a single spin state by removing the other via a resonant spin-removal laser [23].

\section{SHORT-TIME DENSITY DYNAMICS}

To obtain a density distribution of the hole location, we average the singles density distribution at a given time $\tau$. We then subtract it from an average background obtained without the hole. This process allows us to first cancel out density fluctuations in the form of doublon-hole pairs, which are imaged as empty sites, and, second, to remove residual systematic offsets in the particle density that may result from lattice inhomogeneities [21]. For an enhanced data collection rate at times $\tau<\hbar / t$, we simultaneously study four holes arranged in a seven-site-wide square pattern, instead of a single central hole [Fig. 1(b)]. The density on the pinned sites initially ranges from $0.71(5)$ to $0.921(27)$ without significantly affecting their adjacent sites [21].

The resultant hole density distributions at short times are shown in Fig. 2(a). Within half a tunneling period, the hole tunnels to the four neighboring sites $[\tau=0.47(1) \hbar / t]$; its subsequent propagation retains clear coherent features such as the oscillation of the hole density on the sites adjacent to the origin [maximal at $\tau=0.47(2) \hbar / t$ ], which sets it apart from a classical diffusion process.

We can gain microscopic insights on the role of spin by plotting the hole densities on the central site and its diagonal sites, as shown in Fig. 2(b), at times shorter than the spin superexchange timescale $\hbar / J=2.18(7) \hbar / t$. Within two tunneling periods, hole motion is affected by the presence or absence of quantum interference effects between the two paths leading to the same corner site but possibly distinguishable spin backgrounds. To quantify this effect, we show the predictions of three models that feature different magnetic phases: a noninteracting quantum walk, equivalent to the propagation of a hole in a spin-polarized background; a quantum Monte Carlo (QMC) simulation on a disordered spin background $(T=\infty, J=0)$ [24,25]; and 


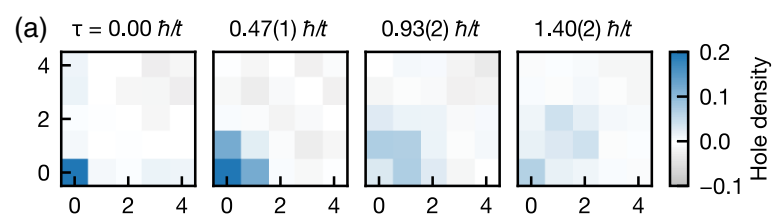

(b)

+ Experiment -- Quantum walk

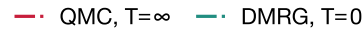
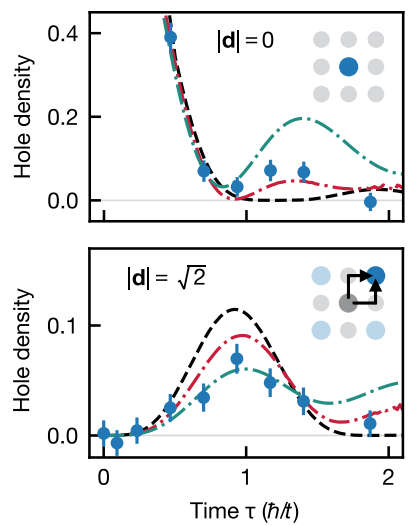

(c) Interfering

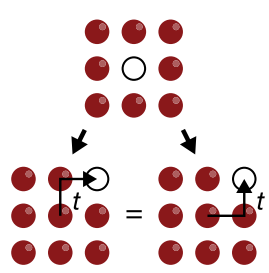

(d) Noninterfering

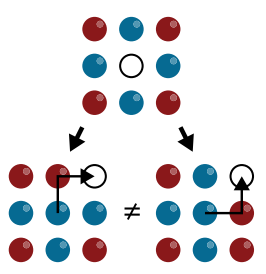

FIG. 2. Effect of quantum interference on short-time density dynamics. (a) Symmetrized average hole densities at short times. The hole initially delocalizes coherently, as indicated by density oscillations on sites surrounding the origin. (b) Short-time evolution of the hole density at the center (distance $|\mathbf{d}|=0$ ) and on the diagonally adjacent sites $(|\mathbf{d}|=\sqrt{2})$. We compare experimental data to models featuring a ferromagnetic (noninteracting quantum walk, dashed black line), disordered (noninteracting, spin-1/2 QMC simulation at $T=\infty$, dash-dotted red line), or antiferromagnetic spin background (ground-state DMRG simulation at $t / J=2$, dash-dotted green line). The diagonal density shows a decreased oscillation amplitude compared to the quantum walk and QMC simulations. This can be interpreted as a manifestation of quantum interference between two nonequivalent hole paths, maximal for a polarized spin background (c) and reduced for an antiferromagnet (d). Here and in the following, error bars indicate a one-sigma statistical uncertainty in the plotted values.

a time-dependent density matrix renormalization group (TD-DMRG) simulation of the $t-J$ model with $t / J=2$ on an $18 \times 4$ system, initially in the antiferromagnetic ground state [26]. All three models quantitatively describe the experimental results during the first tunneling event up to $\tau \approx 0.5 \hbar / t$; a detailed comparison is presented in Ref. [21]. Afterwards, the spinful simulations predict a revival of the central density around $\tau \approx 1.2 \hbar / t$, though finite-size effects magnify this revival in the TD-DMRG simulation. The amplitude of the oscillation in the diagonal density is directly related to the indistinguishability of spin backgrounds after two tunneling events ending at a given site, as sketched in Figs. 2(c) and 2(d): Quantum interference is maximal in a ferromagnet (free quantum walk), reduced in an antiferromagnet (ground-state TDDMRG), and between these two extremes in a paramagnet

(infinite-temperature QMC). The suppressed diagonal density seen experimentally at $\tau \approx 1 \hbar / t$ therefore hints at the quantum statistical role of the antiferromagnetic background in the hole dynamics over short timescales.

\section{LONG-TIME HOLE DELOCALIZATION}

The previous experimental data focus on times smaller than the spin exchange time $\hbar / J$, where the hole motion is essentially driven by direct tunneling. Investigating dynamics at longer times is challenging, as it requires resolving hole densities inversely proportional to the square of its distance to the origin. To mitigate statistical fluctuations in the hole density at large distances, we fit the experimental data in an analysis region containing most of the hole density to a two-dimensional Gaussian distribution that empirically match the hole distribution at longer times [21]. The delocalization dynamics is then quantitatively described by the root-mean-square (rms) hole distance:

$$
d_{\mathrm{rms}}=\sqrt{\sum_{d_{x}, d_{y}}\left(d_{x}^{2}+d_{y}^{2}\right) \rho_{\mathbf{d}} / \sum_{d_{x}, d_{y}} \rho_{\mathbf{d}}},
$$

where $\rho_{\mathbf{d}}$ is the fitted hole density at coordinate $\mathbf{d}=\left(d_{x}, d_{y}\right)$ relative to the initial hole position.

We plot the rms distance of the fitted distribution in Fig. 3. At times $\tau<1 \hbar / t$, we observe a fast linear growth characteristic of a ballistic expansion. The data match the analytical expression for a noninteracting quantum walk, $d_{\text {rms }}=v \tau$ with velocity $v=2 t / \hbar$, confirming that the spin background can be effectively neglected during the first tunneling event. The delocalization then clearly slows after $\tau=1 \hbar / t$; the hole eventually leaves our analysis region of 11 sites in radius [Fig. 1(b)] at times greater than $\tau=10 \hbar / t[21]$.

We can qualitatively capture this slowdown with an analytical model depending only on $t$, mapping hole motion on a spinful background to a free quantum walk on a Bethe lattice [25,27]. The model predicts a crossover from ballistic to diffusive behavior, resulting from the absence of quantum interference between paths leading to the same hole position but different spin backgrounds [24]. Its rms distance asymptotically follows a squareroot law $d_{\text {rms }}=\sqrt{4 D \tau}$ with a diffusion constant $D \approx$ $1.37 D_{\text {MIR }}=1.37 \mathrm{ta}^{2} / \hbar$ close to the Mott-Ioffe-Regel (MIR) limit, which was experimentally shown to be a lower bound for diffusion at larger doping [16]. A similar ballistic-to-diffusive crossover occurs in spin-1/2 QMC simulations at infinite temperature, with a diffusion constant increased by less than $10 \%$ compared to the Bethe lattice case with effective infinite spin of the Bethe lattice, due to enhanced quantum interference [25].

Though the Bethe-lattice model qualitatively predicts the slowdown of the hole, its rms distance exceeds that of the experiment at long times (with a chi-square value $\chi^{2}=47$ 


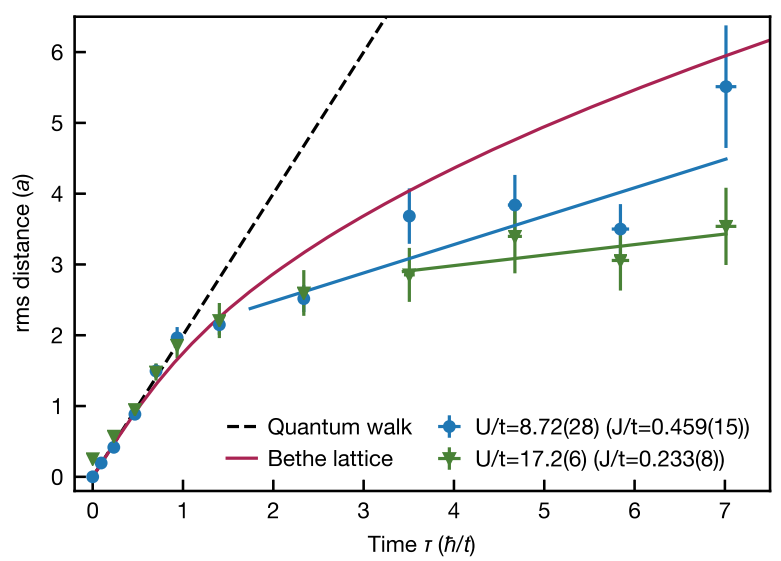

FIG. 3. Long-time slowdown of the hole propagation due to superexchange. Root-mean-squared distance from the origin, obtained from a Gaussian fit to the 2D hole density distribution. The initial linear increase indicates a ballistic expansion compatible with a noninteracting quantum walk (dashed black line). The hole slows down around $\tau=1 \hbar / t$, an effect qualitatively predicted by an analytic model based on a free quantum walk in a Bethe lattice (solid blue line). At later times, the hole is more confined than the Bethe-lattice prediction. Increasing the interaction energy decreases the superexchange and the long-time hole velocity, from $0.40(10) a /(\hbar / t)$ at $U / t=8.72(28)$ to $0.15(17) a /(\hbar / t)$ at $U / t=17.2(6)$ as obtained from linear fits at $\tau \geq 0.8 \hbar / J$.

on the six experimental times $\tau>\hbar / t$ corresponding to a $p$ value of $\left.p=2 \times 10^{-8}\right)$. This deviation challenges the assumption of tunneling being the only relevant energy scale. To directly verify the role of the superexchange on hole motion, we use a Feshbach resonance to double the on-site interaction to $U / t=17.2(6)[t / J=4.30(15)]$ at fixed tunneling. We thereby halve the superexchange energy $J$ while slightly decreasing the temperature $T / t=0.241(18)$ and increasing the correlation length $\xi=2.48(25)$. The experimental rms distance shown in Fig. 3 agrees with the previous data at short times, apart from small initial deviations due to a larger hole preparation infidelity [21]. A linear fit for data at $\tau>0.8 \hbar / J$ indicates a reduction of the long-time hole velocity for smaller $J$ from a value of $0.40(10) a /(\hbar / t)$ at $U / t=8.72(28)$ down to $0.15(17) a /(\hbar / t)$ at $U / t=17.2(6)$. These results are consistent with the expected decrease of the quasiparticle bandwidth with spin exchange in the $t$ - $J$ model in the strong-coupling limit $t \gg J$ [5], and are quantitatively compatible with the numerical predictions in [6,28-31]. This agreement suggests that, at long times, the hole becomes dressed by the magnetic background and forms a magnetic polaron.

\section{SPIN DYNAMICS}

The two-stage dynamics of the hole motion are also visible in the spin correlations, whose fast evolution away from equilibrium and slow return to it can be interpreted as the formation and departure of a polaron, respectively. We measure the sign-corrected spin correlation function $C_{\mathbf{r}}(\mathbf{d})=(-1)^{d_{x}+d_{y}} 4\left(\left\langle S_{\mathbf{r}}^{z} S_{\mathbf{r}+\mathbf{d}}^{z}\right\rangle-\left\langle S_{\mathbf{r}}^{z}\right\rangle\left\langle S_{\mathbf{r}+\mathbf{d}}^{z}\right\rangle\right)$ in an independent data set starting with a single hole at the center [21]. We plot a map of the correlations from the initial hole location $C_{\mathbf{0}}(\mathbf{d})$ (as the hole becomes indistinguishable from hole fluctuations once it starts moving) and averaged over all spatial symmetries for select times; see Fig. 4(a). At $\tau=0$, these correlations are vanishing because of the presence of the hole; within one tunneling time, the hole hops to a neighboring site, and the correlations become those of the exchanged neighboring spin [Fig. 4(b)]. This swapping of correlations results in a reversal of the global antiferromagnetic correlation pattern [light brown color in Fig. 4(a)]: For instance, the negative sign of the diagonal correlation $C_{\mathbf{0}}(\mathbf{d}=(1,1))$ at $\tau=0.467(8) \hbar / t$ is at odds with its value at long times, $\tau=23.4(4) \hbar / t$. Here, the reversal of the antiferromagnetic pattern extends up to three sites away from the center and is a dynamical analog to the short-range polaronic behavior seen at equilibrium in Ref. [19]. In contrast, we find that the nearest-neighbor correlator does not exhibit the same sign-change effect [light green squares at $\tau=0.467(8) \hbar / t$ ] as it results from a mixture of diagonal correlations weakened by the presence of the hole before the initial quench.

The swapping mechanism described in Fig. 4(b) suggests a practical way to quantitatively predict the evolution

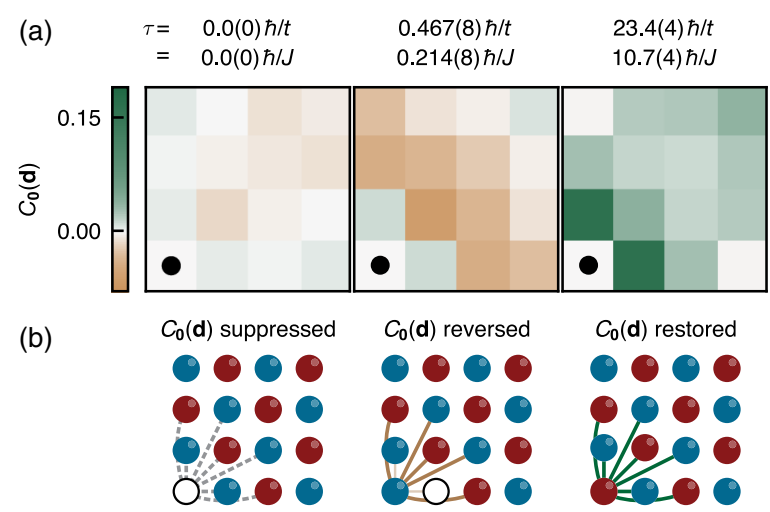

FIG. 4. Reversal and recovery of antiferromagnetic correlations. (a) Sign-corrected spin correlations $C_{\mathbf{0}}(\mathbf{d})$ from the initial hole location (black circle) at select times, symmetrized across reflections. Within one tunneling time, we observe antiferromagnetic correlations but with the opposite sign (light brown), apart from the nearest-neighbor correlator. The correct AFM pattern (green) then slowly restores itself, although the correlations have not fully equilibrated even at our longest measured times, $\tau=23.4(4) \hbar / t=10.7(4) \hbar / J$ [21]. (b) The emergence of shorttime antiferromagnetic correlations with the opposite sign can be understood in a classical Néel state. Initially, the hole swaps a neighboring spin to the origin, producing reversed correlations at the origin site. At later times, relaxation processes restore the correct antiferromagnetism. 
of spin correlations resulting from fast hole dynamics. To do so, we take experimental pictures at $\tau=0$ and displace spins according to the distribution of the hole trajectories predicted by the analytical Bethe-lattice model [21]. In Fig. 5(a), we compare the time evolution of the next-nearest correlations to the center $C_{\mathbf{0}}(|\mathbf{d}|=\sqrt{2})$ with the predicted evolution based on this model, shown as a purple-gray band. It agrees with the experimental data at short times only, indicating that local correlation swapping is an accurate picture in that regime.

A more complete model for the hole dynamics is one that considers the energy cost of correlation swapping, which, at first, binds the hole to around its initial location [32]. Spin exchange can then enable the restoration of the disrupted spin background, leading to a polaron

(a)
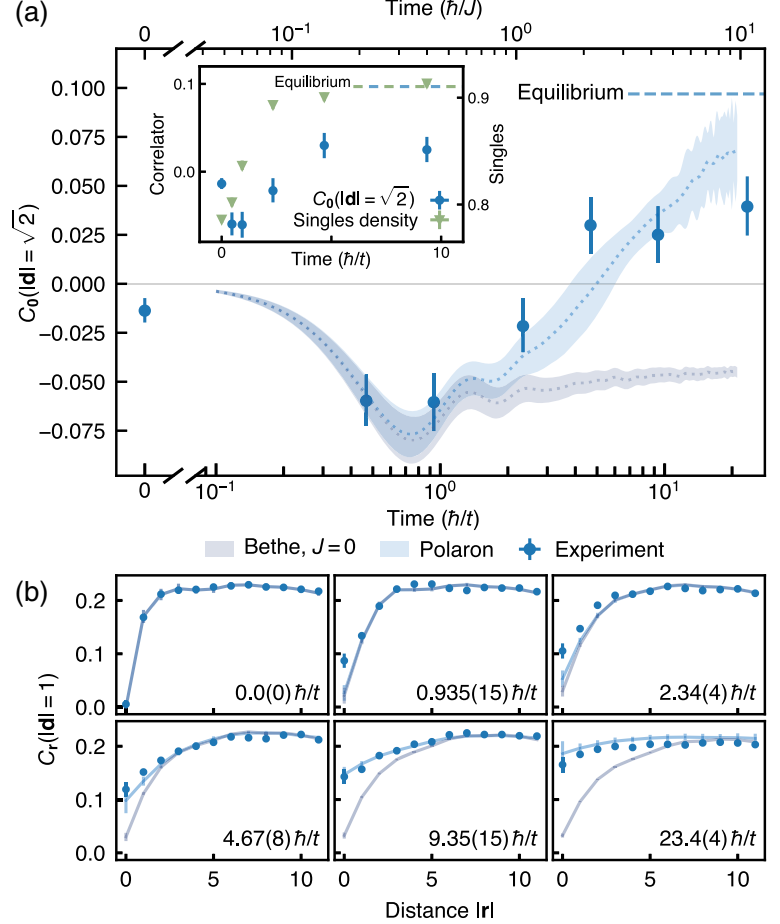

FIG. 5. Comparison with a polaron model. (a) Sign-corrected spin correlations between the initial hole location and its diagonal neighbors $\left[C_{\mathbf{0}}(|\mathbf{d}|=\sqrt{2})\right]$ as a function of time. The short-time dynamics are captured by a Bethe-lattice model considering only charge motion (purple-gray band), while spin exchange must be taken explicitly into account (here, as a polaron model, blue band) to qualitatively describe the long-time relaxation of the correlations towards their equilibrium values (blue dashed line). This correlation relaxation is slower than the density relaxation averaged on a $3 \times 3$ area around the center (inset). (b) Nearest-neighbor correlations as a function of distance to the center $C_{\mathbf{r}}(|\mathbf{d}|=1)$. The slow spreading of the depleted correlations towards the system edges is best captured by the polaron model (blue) as opposed to the Bethe-lattice model (purple-gray). Neither model quantitatively captures the short-time dynamics at the center $(|\mathbf{r}|=0)$. delocalizing with a long-time velocity set by the superexchange energy. An example of such a model is presented in Ref. [31], where the polaron is described as a composite object formed by a holon and spinon connected by a string of displaced correlations. We include the spinon dynamics by shifting the experimental pictures used in our model (and, therefore, the effective hole origin) according to a ballistically propagating spinon [21]. Including these dynamics results in an accurate prediction of the long-time behavior of the spin correlations, qualitatively capturing their slow return to equilibrium [blue band in Fig. 5(a)]. Spin correlations relax more slowly than the local density [inset of Fig. 5(a)]—strikingly, equilibration in the spin sector has not occurred even for the last measured time of $\tau=$ 23.4(4) $\hbar / t$, although the hole itself has left the system.

The polaron model can also be benchmarked with spin correlations measured across the entire Mott-insulating region. We show in Fig. 5(b) the nearest-neighbor correlations $C_{\mathbf{r}}(|\mathbf{d}|=1)$ averaged according to the bond distance to the center $|\mathbf{r}|$ (full data shown in Ref. [21]). Strongly depleted correlations are initially observed around the center due to the hole, before spreading towards larger distances as the system relaxes. Since nearest-neighbor correlations are directly proportional to the local magnetic energy in the $t-J$ approximation, this propagation indicates that the hole imparts its kinetic energy to the entire spin background while leaving the systems.

Including spinon-holon dynamics [solid lines in Fig. 5(b)] compared to a bare Bethe-lattice model (dashed lines) leads to good agreement with the experimental data even at longer times, confirming the role of spin exchange in the long-time dynamics. However, theory underestimates nearest-neighbor correlations at short distances and short times, $\tau=0.94$ and $2.35 \hbar / t$, perhaps as a result of spin relaxation mechanisms not captured in the model, such as magnon emission. Furthermore, we note that the model overestimates the rms distance of the hole measured experimentally [21], possibly because it neglects spin fluctuations that effectively lead to a disordered magnetic background and inhibit hole motion.

In this work, we demonstrate the intricate linkage between charge and spin dynamics in the two-dimensional Fermi-Hubbard model and explore the formation and motion of magnetic polarons. The size, coherence, and control of this cold-atom quantum simulator are key to benchmarking nonequilibrium theories of magnetic polaron formation and numerical techniques away from the linear-response regime. Our work could be extended to investigate the interaction between multiple, deterministically created polarons and to study how magnetism facilitates this interaction. This extension may enable the microscopic observation of $d$-wave Cooper pairs and of emergent phases such as stripe phases, pseudogap phases, and ultimately $d$-wave superconducting phases. 


\section{ACKNOWLEDGMENTS}

We thank E. Altman, J. Carlström, M. Kanász-Nagy, J. Léonard, M. Knap, A. Lukin, E. Manousakis, Z.-X. Shen, and W. Zwerger for insightful discussions. We acknowledge support from the Gordon and Betty Moore Foundation Grant No. 6791; NSF Grants No. PHY-1734011, No. OAC-1934598, and No. OAC-1934714; ONR Grants No. W911NF-11-1-0400 and No. N00014-18-1-2863; ARO Grant No. W911NF-20-1-0163; ARO/AFOSR/ONR DURIP; the U.S. Department of Defense through the NDSEG program (G. J.), the NSF GRFP (L.H.K. and C.S.C.), the Deutsche Forschungsgemeinschaft (DFG, German Research Foundation) under Germany's Excellence Strategy-EXC-2111-390814868 (A. B. and F. G.), the Swiss National Science Foundation, and the Max Planck/Harvard Research Center for Quantum Optics (M. L.).

[1] W. F. Brinkman and T. M. Rice, Single-Particle Excitations in Magnetic Insulators, Phys. Rev. B 2, 1324 (1970).

[2] É. L. Nagaev, Ferromagnetic and Antiferromagnetic Semiconductors, Sov. Phys. Usp. 18, 863 (1975).

[3] S. Schmitt-Rink, C. M. Varma, and A. E. Ruckenstein, Spectral Function of Holes in a Quantum Antiferromagnet, Phys. Rev. Lett. 60, 2793 (1988).

[4] C. L. Kane, P. A. Lee, and N. Read, Motion of a Single Hole in a Quantum Antiferromagnet, Phys. Rev. B 39, 6880 (1989).

[5] S. Sachdev, Hole Motion in a Quantum Néel State, Phys. Rev. B 39, 12232 (1989).

[6] Z. Liu and E. Manousakis, Dynamical Properties of a Hole in a Heisenberg Antiferromagnet, Phys. Rev. B 45, 2425 (1992).

[7] E. Dagotto, Correlated Electrons in High-Temperature Superconductors, Rev. Mod. Phys. 66, 763 (1994).

[8] A. S. Mishchenko and N. Nagaosa, Electron-Phonon Coupling and a Polaron in the $t$-J Model: From the Weak to the Strong Coupling Regime, Phys. Rev. Lett. 93, 036402 (2004).

[9] E. Manousakis, String Excitations of a Hole in a Quantum Antiferromagnet and Photoelectron Spectroscopy, Phys. Rev. B 75, 035106 (2007).

[10] A. Damascelli, Z. Hussain, and Z.-X. Shen, Angle-Resolved Photoemission Studies of the Cuprate Superconductors, Rev. Mod. Phys. 75, 473 (2003).

[11] M. Cetina, M. Jag, R. S. Lous, I. Fritsche, J. T. M. Walraven, R. Grimm, J. Levinsen, M. M. Parish, R. Schmidt, M. Knap, and E. Demler, Ultrafast Many-Body Interferometry of Impurities Coupled to a Fermi Sea, Science 354, 96 (2016).

[12] C. Weitenberg, M. Endres, J. F. Sherson, M. Cheneau, P. Schauß, T. Fukuhara, I. Bloch, and S. Kuhr, Single-Spin Addressing in an Atomic Mott Insulator, Nature (London) 471, 319 (2011).
[13] P. M. Preiss, R. Ma, M. E. Tai, A. Lukin, M. Rispoli, P. Zupancic, Y. Lahini, R. Islam, and M. Greiner, Strongly Correlated Quantum Walks in Optical Lattices, Science 347, 1229 (2015).

[14] J. Vijayan, P. Sompet, G. Salomon, J. Koepsell, S. Hirthe, A. Bohrdt, F. Grusdt, I. Bloch, and C. Gross, Time-Resolved Observation of Spin-Charge Deconfinement in Fermionic Hubbard Chains, Science 367, 186 (2020).

[15] R. Anderson, F. Wang, P. Xu, V. Venu, S. Trotzky, F. Chevy, and J. H. Thywissen, Conductivity Spectrum of Ultracold Atoms in an Optical Lattice, Phys. Rev. Lett. 122, 153602 (2019).

[16] P. T. Brown, D. Mitra, E. Guardado-Sanchez, R. Nourafkan, A. Reymbaut, C.-D. Hébert, S. Bergeron, A.-M. S. Tremblay, J. Kokalj, D. A. Huse, P. Schauß, and W. S. Bakr, Bad Metallic Transport in a Cold Atom FermiHubbard System, Science 363, 379 (2019).

[17] M. A. Nichols, L. W. Cheuk, M. Okan, T. R. Hartke, E. Mendez, T. Senthil, E. Khatami, H. Zhang, and M. W. Zwierlein, Spin Transport in a Mott Insulator of Ultracold Fermions, Science 363, 383 (2019).

[18] C. S. Chiu, G. Ji, A. Bohrdt, M. Xu, M. Knap, E. Demler, F. Grusdt, M. Greiner, and D. Greif, String Patterns in the Doped Hubbard Model, Science 365, 251 (2019).

[19] J. Koepsell, J. Vijayan, P. Sompet, F. Grusdt, T. A. Hilker, E. Demler, G. Salomon, I. Bloch, and C. Gross, Imaging Magnetic Polarons in the Doped Fermi-Hubbard Model, Nature (London) 572, 358 (2019).

[20] C. S. Chiu, G. Ji, A. Mazurenko, D. Greif, and M. Greiner, Quantum State Engineering of a Hubbard System with Ultracold Fermions, Phys. Rev. Lett. 120, 243201 (2018).

[21] See Supplemental Material at http://link.aps.org/ supplemental/10.1103/PhysRevX.11.021022 for a description of the experimental methods, data analysis, numerical simulations, and spinon-holon model.

[22] A. Mazurenko, C. S. Chiu, G. Ji, M. F. Parsons, M. KanászNagy, R. Schmidt, F. Grusdt, E. Demler, D. Greif, and M. Greiner, A Cold-Atom Fermi-Hubbard Antiferromagnet, Nature (London) 545, 462 (2017).

[23] M. F. Parsons, A. Mazurenko, C. S. Chiu, G. Ji, D. Greif, and M. Greiner, Site-Resolved Measurement of the SpinCorrelation Function in the Fermi-Hubbard Model, Science 353, 1253 (2016).

[24] J. Carlström, N. Prokof'ev, and B. Svistunov, Quantum Walk in Degenerate Spin Environments, Phys. Rev. Lett. 116, 247202 (2016).

[25] M. Kanász-Nagy, I. Lovas, F. Grusdt, D. Greif, M. Greiner, and E. A. Demler, Quantum Correlations at Infinite Temperature: The Dynamical Nagaoka Effect, Phys. Rev. B 96, 014303 (2017).

[26] A. Bohrdt, F. Grusdt, and M. Knap, Dynamical Formation of a Magnetic Polaron in a Two-Dimensional Quantum Antiferromagnet, New J. Phys. 22, 123023 (2020).

[27] D. Golež, J. Bonča, M. Mierzejewski, and L. Vidmar, Mechanism of Ultrafast Relaxation of a Photo-Carrier in Antiferromagnetic Spin Background, Phys. Rev. B 89, 165118 (2014). 
[28] E. Dagotto, R. Joynt, A. Moreo, S. Bacci, and E. Gagliano, Strongly Correlated Electronic Systems with One Hole: Dynamical Properties, Phys. Rev. B 41, 9049 (1990).

[29] G. Martinez and P. Horsch, Spin Polarons in the t-J Model, Phys. Rev. B 44, 317 (1991).

[30] P. W. Leung and R. J. Gooding, Dynamical Properties of the Single-Hole t-J Model on a 32-Site Square Lattice, Phys. Rev. B 52, R15711 (1995).
[31] F. Grusdt, A. Bohrdt, and E. Demler, Microscopic SpinonChargon Theory of Magnetic Polarons in the t-J Model, Phys. Rev. B 99, 224422 (2019).

[32] L. N. Bulaevskii, E. L. Nagaev, and D. I. Khomskii, A New Type of Auto-localized State of a Conduction Electron in an Antiferromagnetic Semiconductor, J. Exp. Theor. Phys. 27, 836 (g1968), http://www.jetp.ac.ru/cgi-bin/e/index/e/27/5/ p836? $\mathrm{a}=$ list. 